\title{
Reparación endovascular en un paciente con aneurisma de aorta abdominal y riñón en herradura. Reporte de caso \\ y revisión de la literatura
}

\author{
Patricio David Gavilanes Carrasco \\ pdgavilanes@puce.edu.ec \\ https://orcid.org/0000-0001-6566-1844 \\ Pontificia Universidad Católica del Ecuador \\ Evelyn Cristina Carrera Garrido \\ eccg1617@gmail.com \\ https://orcid.org/0000-0002-6465-4404 \\ Universidad Central del Ecuador \\ Ernesto Sebastián Padilla Yánez \\ sebastianpadillayanez@gmail.com \\ https://orcid.org/0000-0003-1000-2408 \\ Postgrado Pontificia Universidad Católica del Ecuador \\ Ruddy Lisseth Ruiz Segarra \\ ruddy.ruiz4@hotmail.com \\ https://orcid.org/0000-0003-0341-368X \\ Hospital General Provincial Pablo Arturo Suárez \\ Lisseth Stefanía Ramírez Velasco \\ liss1994chete@outlook.com \\ https://orcid.org/0000-0003-4609-9029?lang=en \\ Hospital Alfredo Noboa Montenegro \\ Quito - Ecuador.
}

\section{RESUMEN}

Objetivo: Demostrar que el tratamiento endovascular es la mejor opción terapéutica en pacientes con aneurisma de aorta abdominal y riñón en herradura. Además de revisar que estas patologías asociadas son poco frecuentes, encontrándose en el $0.12 \%$ de los pacientes tratados. Esta asociación incrementa la dificultad técnica de reparación abierta. Descripción del caso: Se analiza a una paciente femenina adulta mayor que acude a centro particular por dolor abdominal, al examen físico: abdomen distendido, doloroso a la palpación en epigastrio y mesogastrio con masa pulsátil, se realiza tomografía abdominal evidenciándose incidentalmente un aneurisma de aorta abdominal y riñón en 
herradura por lo que se solicitó valoración por el servicio de cirugía vascular. En conclusión: El tratamiento endovascular del aneurisma de aorta abdominal se presenta como la mejor alternativa en pacientes con ausencia de insuficiencia renal, anatomía favorable y arterias accesorias renales menores a $3 \mathrm{~mm}$.

Palabras clave: aneurisma de aorta abdominal; riñón en herradura; reparación endovascular de aneurisma. 


\title{
Endovascular repair in a patient with abdominal aortic aneurysm and horseshoe kidney. Case report and literature review
}

\begin{abstract}
Objective: To demonstrate that endovascular treatment is the best therapeutic option in patients with abdominal aortic aneurysm and horseshoe kidney. In addition to reviewing that these associated pathologies are rare, being found in $0.12 \%$ of treated patients. This association increases the technical difficulty of open repair. Description of the case: An elderly female patient who attends a private center due to abdominal pain is analyzed. On physical examination: distended abdomen, painful on palpation in the epigastrium and mesogastrium with a pulsatile mass. Abdominal tomography is performed, incidentally evidencing an abdominal aortic aneurysm. and horseshoe kidney, for which assessment was requested by the vascular surgery service. In conclusion: Endovascular treatment of abdominal aortic aneurysm is presented as the best alternative in patients with no renal insufficiency, favorable anatomy and renal accessory arteries smaller than $3 \mathrm{~mm}$.
\end{abstract}

Keywords: abdominal aortic aneurysm; horseshoe kidney; endovascular aneurysm repair.

Artículo recibido: 02 enero 2022 Aceptado para publicación: 28 enero 2022 Correspondencia: pdgavilanes@puce.edu.ec Conflictos de Interés: Ninguna que declarar 


\section{INTRODUCCIÓN.}

El riñón en herradura es una de las malformaciones renales más frecuentes y suele cursar con función renal normal, con un predominio en el sexo masculino de 2 a 1. (Senarriaga et al., 2009).

Con respecto a la edad no hay diferencias entre hombres y mujeres. En las mujeres se encontró más frecuentemente antecedentes de hipertensión, diabetes mellitus, dislipidemia y enfermedad renal crónica que en los hombres. En promedio de los tiempos operatorios para fumadores fueron más altos que para los no fumadores. (Gonzalez-

\section{Urquijo et al, 2021)}

Esta ocasionado por la fusión de dos masas renales, a nivel del polo inferior en un $95 \%$, situadas verticalmente, que suelen localizarse a nivel de la bifurcación aórtica. La vascularización anormal y la presencia de arterias accesorias se da en un $80 \%$ de los casos, cerca del $30 \%$ presenta 1 arteria renal, $70 \%, 2$ o 3 arterias, que difieren en su origen y diámetro. Su etiología es desconocida. A nivel embrionario, esta anomalía se produce entre la 4 y 6 semana de gestación. La alteración de la posición de los vasos umbilicales, pelvianos, mesentéricos superiores y el desarrollo anómalo de la pelvis renal podrían determinar que se fusionen (Merino et al., 2012).

Se asocia a 1- 5/1.000 aneurismas de aorta abdominal que precisan corrección quirúrgica. La presencia del AAA y el riñón en herradura, aumenta la dificultad técnica en la cirugía convencional, sea hecha vía transperitoneal o extraperitoneal, las complicaciones postquirúrgicas se incrementan (Kaplan DB, Kwon CC, Marin ML, Hollier LH, 1999). La tomografía computarizada (TC) es la principal prueba diagnóstica. La corrección endovascular se ha convertido en un abordaje común para el reparo de AAA y representa una alternativa válida en pacientes seleccionados con AAA y anomalías renales congénitas concomitantes (Saadi EK, Dussin LH, Moura L, Zago AJ, 2008).

\section{METODOLOGÍA.}

En el presente reporte de caso se circunscribe a un estudio de tipo observacional descriptivo basado en el tratamiento en pacientes con coexistencia de aneurisma de aorta abdominal (AAA) y riñón en herradura. Para esto se realizó la búsqueda bibliográfica y análisis del tratamiento en este tipo de pacientes con la reparación endovascular.

\section{PRESENTACIÓN DE CASO.}

Paciente femenina con aneurisma de aorta abdominal y riñón en herradura que fue intervenida en nuestro hospital mediante EVAR. 
Se realizó la búsqueda de la literatura en Pubmed/ Medline, mediante los términos: Horseshoe Kidney, endovascular, aneurysm, y EVAR. Nosotros identificamos 25 casos en la base de datos desde 1997 hasta la fecha actual. (Tabla 1)

Tabla 1. Casos reportados de asociación de pacientes con riñón en herradura y aneurisma de aorta abdominal intervenidos con tratamiento endovascular.

\begin{tabular}{|c|c|c|c|c|c|c|c|c|}
\hline $\mathrm{N} \cdot$ & Año & Autores & $\begin{array}{l}\text { Edad } \\
\text { años }\end{array}$ & Sexo & $\begin{array}{c}\text { Diámetro } \\
\mathrm{mm}\end{array}$ & Arterias Renales & $\begin{array}{l}\text { Clasificación } \\
\text { Elsendrath }\end{array}$ & $\begin{array}{l}\text { Elevación de } \\
\text { creatinina } \\
\text { preoperatoria }\end{array}$ \\
\hline 1 & 1997 & Ferko et al. & 86 & Varón & - & $2+1$ & 11 & No \\
\hline 2 & 1998 & Dorffner et al. & - & Varón & - & - & - & No \\
\hline 3 & 1998 & Loftus et al. & 59 & Varón & 59 & $2+1$ & 11 & No \\
\hline 4 & & & 73 & Varón & 63 & 2 & 1 & No \\
\hline 5 & 1999 & Kaplan et al. & - & Varón & 61 & $3+4$ & iv & No \\
\hline 6 & 2001 & Lee et al. & 78 & Varón & 52 & $2+1$ & 11 & No \\
\hline 7 & 2001 & Toursarkissian et al. & 70 & Varón & 50 & $2+4$ & iv & Transitoria \\
\hline 8 & 2003 & Teljink et al. & 78 & Varón & 72 & 2 & 1 & No \\
\hline 9 & 2003 & Ruppert et al & 70 & Varón & 58 & $2+1$ & $\|$ & No \\
\hline 10 & & & 70 & Varón & 58 & 2 & 1 & No \\
\hline 11 & & & 69 & Varón & 65 & $2+1$ & II & No \\
\hline 12 & 2004 & Jackson et al. & - & Varón & 59 & $2(1-4)$ & - & Transitoria \\
\hline 13 & & & - & Varón & - & $2(1-4)$ & - & Transitoria \\
\hline 14 & & & - & Varón & - & $2(1-4)$ & - & No \\
\hline 15 & & & - & Varón & - & $2(1-4)$ & - & No \\
\hline 16 & 2005 & Volpe et al. & 65 & Varón & 50 & $2+2$ & - & Transitoria \\
\hline 17 & 2006 & sajid et al. & 80 & Mujer & 63 & $2+2$ & - & No \\
\hline 18 & 2008 & Saadi et al. & $6 B$ & Mujer & $6 B$ & $2+3$ & - & Transitoria \\
\hline 19 & 2009 & Brechtel et al. & 78 & Varón & 51 & $2+1$ & III & No \\
\hline 20 & 2011 & Merino et al. & 60 & Mujer & 62 & $2+2$ & IV & Transitoria \\
\hline 21 & & & 71 & Mujer & 47 & $2+1$ & ॥ & No \\
\hline 22 & 2012 & Krivoshei et al. & 81 & Varón & 58 & $2+2$ & IV & No \\
\hline 23 & 2014 & Brown et al. & 58 & Varón & 42 & $2+1$ & 11 & No \\
\hline 24 & & & 82 & Varón & 49 & $2+1$ & II & No \\
\hline 25 & & & 66 & Varón & 54 & 2 & 1 & si \\
\hline
\end{tabular}

\section{Caso clínico}

Se trata de una paciente femenina de 71 años de edad, con antecedentes de hipertensión arterial y gastritis crónica, que presentó cuadro de 8 días de evolución de dolor abdominal tipo transfictivo localizado en epigastrio con irradiación a la espalda de moderada intensidad. Fue atendida en un centro particular donde realizaron tomografía abdominal que evidenció incidentalmente un aneurisma de aorta abdominal y motivó su transferencia al servicio de cirugía vascular.

A su llegada presenta los siguientes signos vitales: TA 146/100 mmHg; FC: 77 por min; FR: 20 por min. Al examen físico: Cardiopulmonar: normal. Abdomen: distendido, doloroso a la palpación en epigastrio y mesogastrio, con masa pulsátil a este nivel. Pulsos tronculares y distales presentes. Laboratorios: Creatinina de $0.8 \mathrm{mg} / \mathrm{dl}$. 
Figura 1. Aneurisma de aorta abdominal asociado a riñón en herradura.

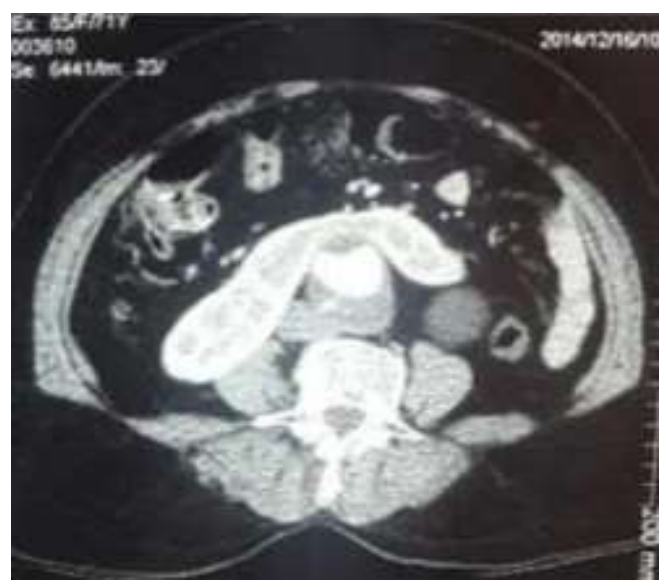

Se inició inmediatamente tratamiento antihipertensivo con atenolol y se realizó de forma urgente una angiotomografía que reveló un aneurisma de aorta abdominal infra renal fusiforme de $7.53 \mathrm{~cm} \times 6.5 \mathrm{~cm}$, con trombo mural y placa de ateroma. (Fig.1) El aneurisma inicia a $4.07 \mathrm{~cm}$ de la arteria renal derecha, con una longitud de $10.6 \mathrm{~cm}$, que se extiende a la arteria iliaca primitiva izquierda, hasta la bifurcación con diámetro de 1.7 cm a nivel de iliaca interna. (Fig. 2). Hallazgo asociado con riñón en herradura tipo II (según la clasificación de Eisendrath).

Figura 2. Reconstrucción tomográfica y visualización de las arterias renales

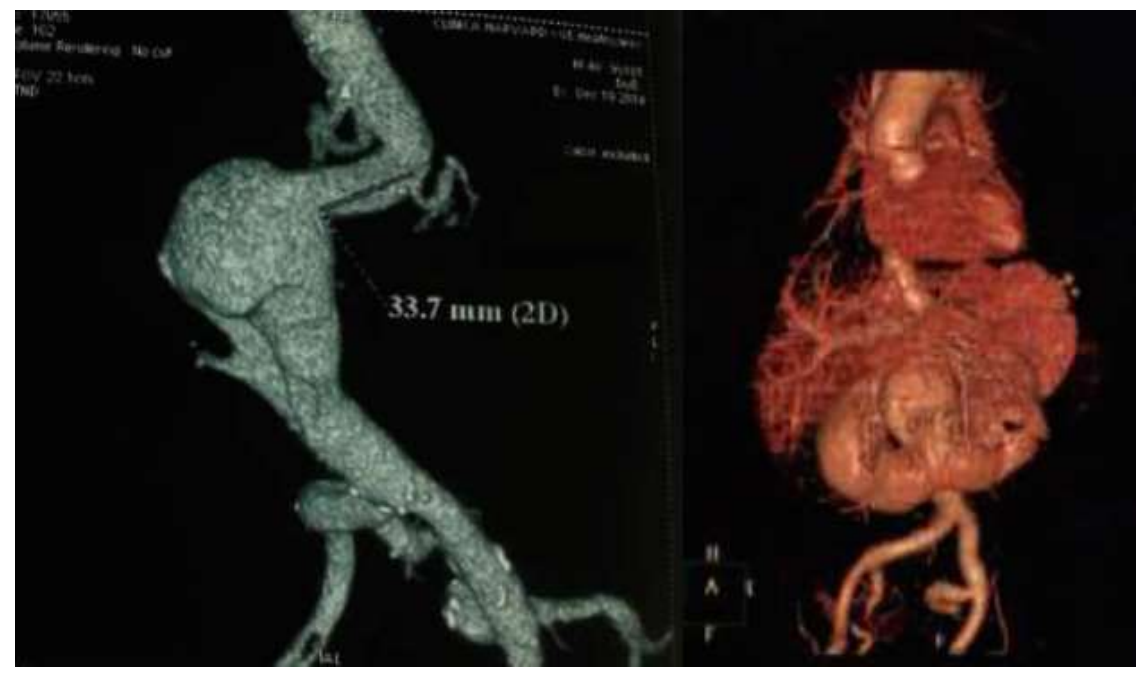

\section{RESULTADOS}

El caso fue tratado: con el paciente bajo anestesia general con corrección endovascular que se realizó en sala de operaciones, ambas arterias femorales fueron disecadas. Se utilizó una endoprótesis Zenith de 24 x 111mm (Cook ZSLE-13-122-ZT Inc.). El cuerpo principal bifurcado Zenith fue colocado y desplegado a través de una guía rígida desde la 
ingle derecha. Posteriormente se desplegó ambas, una extensión iliaca contralateral de 13 x $122 \mathrm{~mm}$ y una rama iliaca ipsilateral de 20 x $74 \mathrm{~mm}$, previa embolización de la arteria hipogástrica izquierda.

Se completó el procedimiento con angiografía de control que reveló la exclusión exitosa del aneurisma sin endofugas. Las arterias renales permanecieron permeables. Se embolizó la arteria hipogástrica izquierda para evitar endofugas. Se retiraron los dispositivos de liberación exitosamente y se realizó cierre de arteriotomías y síntesis por planos.

Figura.3. Tratamiento endovascular de Aneurisma de aorta abdominal

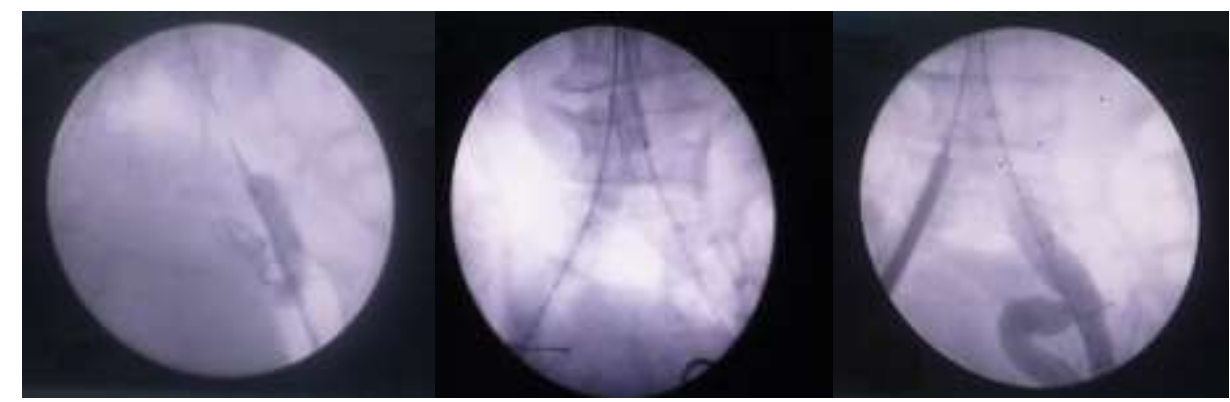

El paciente cursó con evolución satisfactoria en el postquirúrgico en unidad de terapia intensiva, y fue dado el alta hospitalaria al tercer día. Su función renal permaneció estable con nivel de creatinina de $1.0 \mathrm{mg} / \mathrm{dl}$ al alta. Angiotomografía de control y seguimiento a los 6 meses revela que la endoprótesis está en excelente posición; las arterias renales permanecen permeables sin evidencia de endofugas. (Fig,4)

Figura 4. Control post tratamiento endovascular de AAA a los 6 meses.

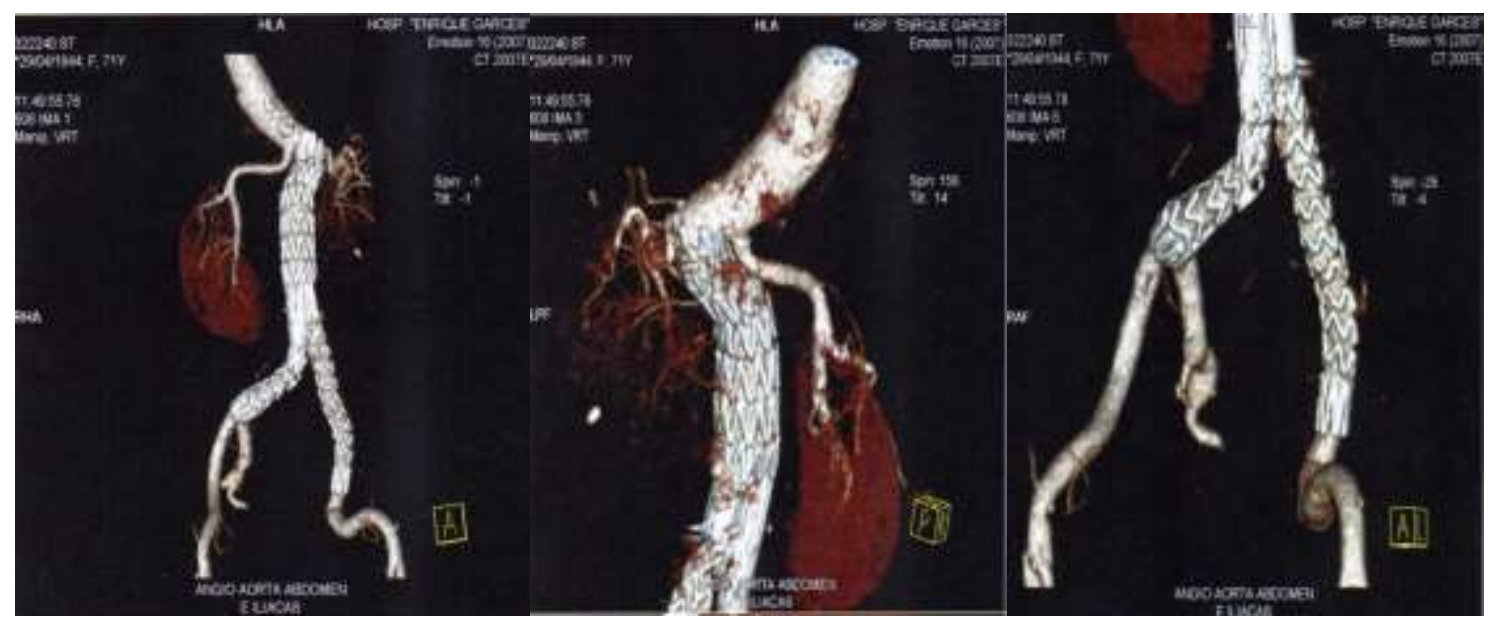

\section{DISCUSIÓN}

Las anomalías renales congénitas en el contexto de cirugía de aorta, presenta desafíos técnicos específicos para el cirujano vascular. El riñón en herradura es la anomalía 
congénita renal más común, que se presenta en $0.12 \%$ de los procedimientos quirúrgicos aórticos (Tan T-W \& Farber A, 2011).

Otras anomalías menos frecuentes incluye la presencia de riñón ectópico, anomalías vasculares renales con arterias renales accesorias o supernumerarias (Yano H, Konagai N, Maeda M, et al., 2003).

El riñón en herradura se caracteriza por fusión medial de los dos riñones anteriormente o posteriormente a la aorta abdominal. En más del $90 \%$ de casos la fusión del parénquima con conexión de las dos pelvis renales, se presenta a nivel de los polos inferiores; sin embargo la fusión renal puede ocurrir a cualquier nivel (Tan T-W \& Farber A, 2011). Esta anomalía renal es usualmente asintomática, asociado con función renal normal y su diagnóstico incidental en exámenes de imagen es muy frecuente. ${ }^{9}$ Aunque existe una variedad de clasificaciones que describen al riñón en herradura, la más usada es la clasificación de Eisendrath (Fig.5) Nuestro paciente tuvo clasificación tipo II, la cuál es susceptible de manejo endovascular (Merino et al., 2012).

Figura 5. Tipos de riñón en herradura atendiendo a la variabilidad en la vascularización. Modificada de Eisendrath

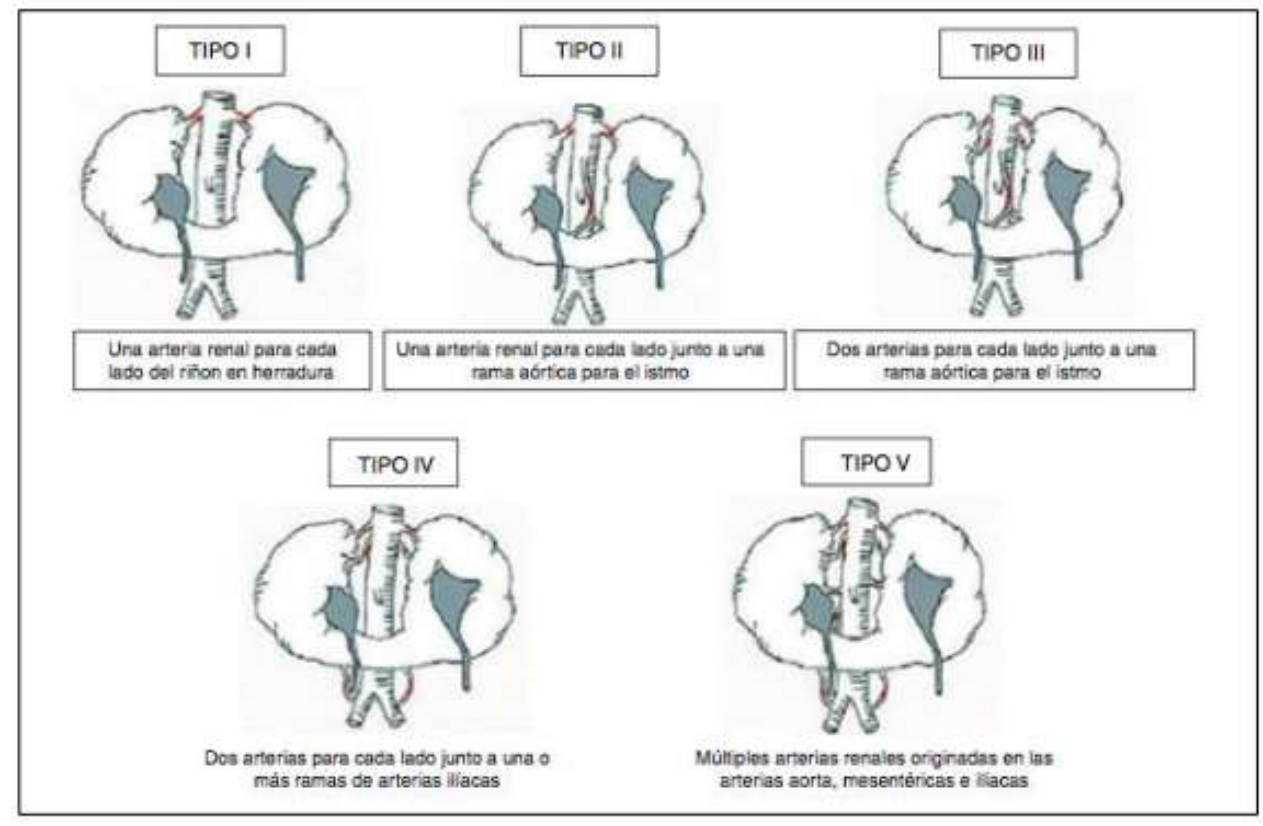

Se ha documentado previamente, dificultades técnicas asociadas a la corrección quirúrgica abierta de AAA, con la coexistencia de riñón en herradura. El primer reto involucra la selección del abordaje quirúrgico. La laparotomía media provee mejor exposición del aneurisma y del riñón, sin embargo la presencia del istmo renal dificulta la exposición y el control proximal aórtico (Ezzet F, Dorazio R, Herzberg R, 1977). 
Existen dos tipos de abordajes para estos casos el transperitoneal y retroperitoneal decritos en tabla 2, las ventajas y desventajas. La diferencia es que el abordaje transperitoneal es más adecuado para casos de emergencia de AAA rotos, porque nos permite un control rápido del cuello del aneurisma. En cambio, un abordaje retroperitoneal izquierdo evita la interferencia con el sistema urinario, de esta manera minimizamos la posibilidad de lesión iatrogénica. (Sachsamanis et al., 2019)

Tabla 2. Abordajes transperitoneal y retroperitoneal en pacientes con aneurisma de aorta y riñón en herradura (HSK)

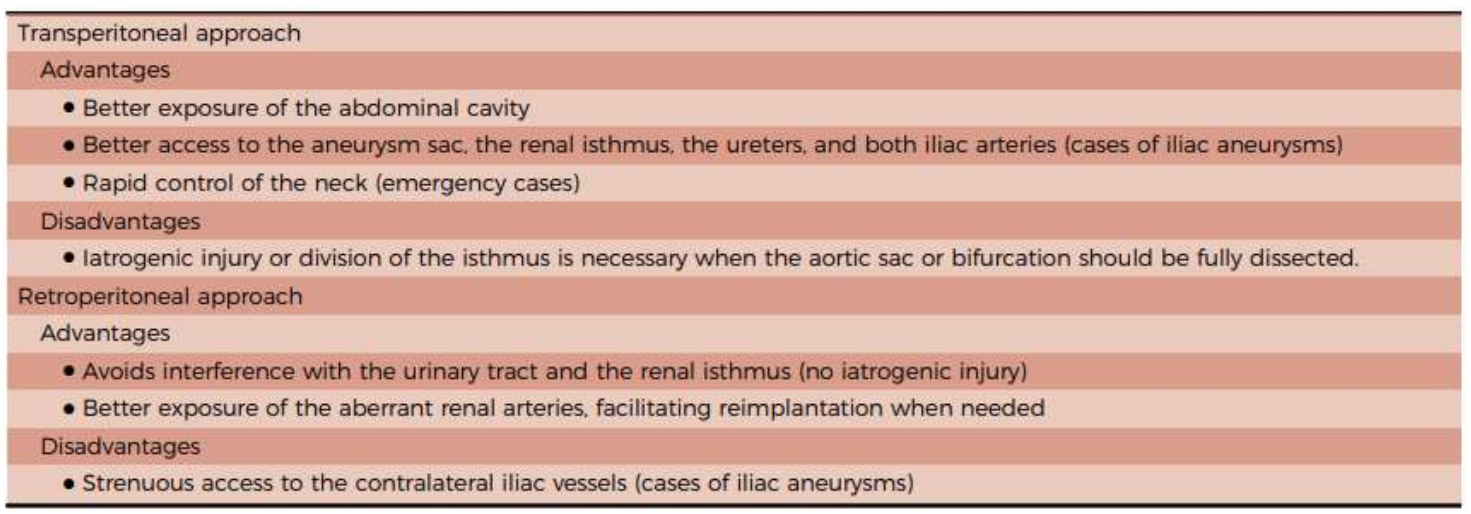

El abordaje retroperitoneal izquierdo se prefiere debido a que se evita manipular el istmo renal y el tracto urinario, sin embargo, es difícil y muy limitado el acceder a la arteria iliaca derecha. El segundo aspecto importante es la decisión de preservar o resecar el istmo renal. Aunque la resección podría ser necesaria para tener una exposición adecuada, el istmo debe ser preservado, si es posible para evitar complicaciones tales como sangrado, y fuga de orina. Finalmente, sobre el 30\% de los pacientes con riñón en herradura tienen una sola arteria renal para cada lado y un ramo adicional que irriga el istmo. Al mismo tiempo pueden identificarse arterias renales que emergen del propio aneurisma. El ligar o reimplantar una arteria renal accesoria que ha menudo se lo hace, es una decisión importante (Stroosma, Kootstra, Schurink, 2001).

La corrección endovascular del AAA ha mostrado estar asociado con menor mortalidad perioperatoria y morbilidad. La técnica EVAR ha demostrado ser segura y eficaz. El primer caso de AAA con asociación a riñón en herradura fue reportado por Ferko en 1997. Desde entonces se han publicado algunos reportes exitosos de corrección endovascular de AAA en pacientes con riñón en herradura. Actualmente los cirujanos determinan la selección de EVAR basados en riesgo clínico y factores anatómicos (Yano et al., 2003). Es evidente, que la técnica EVAR ofrece una ventaja en evitar la complejidad técnica de la corrección abierta del aneurisma. El reto del reparo endovascular de AAA es el de 
manejar las arterias renales aberrantes en el contexto de un riñón en herradura preexistente. Algunos investigadores, sugieren que la oclusión de las arterias renales accesorias durante la corrección endovascular EVAR es segura, especialmente cuando su diámetro es menor de $3 \mathrm{~mm}$ y no existe daño renal preexistente (Brechtel K, Kalender G, Heller S, et al., 2009).

Esta consideración se recomienda en pacientes con AAA y Riñón en herradura Eisendrath tipo I a IV. La angiotomografía es muy útil en el planeamiento preoperatorio para EVAR porque permite la medición exacta de los diámetros aortoiliaco, longitudes y ángulos. Estos parámetros juntos con la determinación de presencia de trombo y enfermedad oclusiva, facilitan la selección apropiada de la endoprótesis y de la estrategia de desplazamiento específica. Es necesario determinar la localización y caracterización del aporte vascular aberrante del riñón en pacientes con anomalías renales tales como riñón en herradura (Brown K, Robinson D, Bray, 2014).

Por último, se debe tomar la decisión de sacrificar una o más arterias renales accesorias y esto dependerá básicamente si se puede obtener una adecuada zona de sellado con la endoprótesis. Se necesita calcular la zona de liberación protésica y tener ángulos apropiados que permitan la correcta visualización de las arterias renales y garantizar una perfecta zona de impactación durante el despliegue (Ruppert et al., 2004).

Para planeamiento EVAR la reconstrucción angiotomográfica o angioresonancia han demostrado ser superior a la angiografía convencional. Sus ventajas incluyen mejor medición de parámetros, reducción del tiempo operatorio, disminución de contraste intravenoso y reducción en la exposición a radiación. El planeamiento exitoso realizado y la selección apropiada de la endoprótesis, permitió la correcta visualización y salvamiento de la arteria renal accesoria (Stroosma, Kootstra, Schurink, 2001).

\section{CONCLUSIÓN}

El tratamiento endovascular es seguro y factible en pacientes cuidadosamente seleccionados con Aneurisma de Aorta Abdominal y con la coexistencia de anomalías renales congénitas. El planeamiento meticuloso preoperatorio es esencial en el manejo endovascular exitoso en estos pacientes.

\section{FINANCIAMIENTO}

Fondo propio de los autores 


\section{REFERENCIAS BIBLIOGRAFICAS}

Senarriaga Ruiz de la Illa N, Vega Manrique R, Lacasa Viscasillas I, Iriarte Soldevilla I, Unda Urzaiz M. (2009). New case of an infrarenal abdominal aortic aneurysm associated with horseshoe kidney. Actas Urol Esp. 33(10):1141-1144.

Illig KA, Green RM. (2001). Diagnosis and management of the 'difficult' abdominal aortic aneurysm: pararenal aneurysms, inflammatory aneurysms, and horseshoe kidney. Semin Vasc Surg 14: 312-7.

Frego M, Bianchera G, Angriman I, Pilon F, Fità C, Miotto D. (2007). Abdominal aortic aneurysm with coexistent horseshoe kidney. Surg Today 37: 626-30.

Merino Díaz B, San Norberto García EM, Martín Pedrosa JM, Revilla Calavia Á, Núñez Miguel E, Vaquero Puerta C. (2012). Tratamiento endovascular de aneurisma de aorta abdominal asociado a riñón en herradura: a propósito de dos casos y revisión de la literatura. Angiologia 64(2):84-91. doi:10.1016/j.angio.2011.11.002.

Kaplan DB, Kwon CC, Marin ML, Hollier LH. (1999). Endovascular repair of abdominal aortic aneurysm in patients with congenital anomalies. J Vasc Surg 30(3):407-15.

Volpe P, Nano G, Dalainas I, Palazzo V, Casana R, Paroni G. (2006). Endovascular repair of an abdominal aortic aneurysm in a patient with horseshoe kidney: Report of a case. Surg Today. 36(7):623-628. doi:10.1007/s00595-006-3213-x.

Saadi EK, Dussin LH, Moura L De, Zago AJ. (2008). Endovascular repair of an abdominal aortic aneurysm in patient with horseshoe kidney: a case report. Rev Bras Cir Cardiovasc. 23(3):425-428.

Lian Krivoshei, Yemi Akin-Olugbade, Glen Mcwilliams, Moshe Halak and Daniel Silverberg. (2012). Endovascular repair of an abdominal aortic aneurysm in the presence of a hydronephrotic horseshoe kidney. Vascular, Vol. 20 No. 1, pp. 5456, Royal Society of Medicine.

Brown K, Robinson D, Bray a. (2014). Customized fenestrated endovascular graft repair of abdominal aortic aneurysm with concomitant horseshoe kidney. Vascular. 22(3):193-197. doi:10.1177/1708538113480577.

Tan T-W, Farber A. (2011). Percutaneous endovascular repair of abdominal aortic aneurysm with coexisting horseshoe kidney: technical aspects and review of the literature. Int J Angiol 20(4):247-50. doi:10.1055/s- 0031-1285105. 
Yano H, Konagai N, Maeda M, et al. Abdominal aortic aneurysm associated with crossed renal ectopia without fusion: case report and literature review. (2003). J Vasc Surg Off Publ Soc Vasc Surg [and] Int Soc Cardiovasc Surgery, North Am Chapter. 37(5):1098- 1102. doi:10.1067/mva.2003.184.

Toursarkissian B, Mejia a, Wholey MH, Lawler M a, Thompson IM, Sykes MT. (2001). Endovascular AAA repair in a patient with a horseshoe kidney and an isthmus mass. J Endovasc Ther. 8(6):604-608. doi:10.1583/1545- 1550(2001)0082.0.CO ;2.

Merino Díaz B, San Norberto García EM, Martín Pedrosa JM, Revilla Calavia Á, Núñez Miguel E, Vaquero Puerta C. (2012). Tratamiento endovascular de aneurisma de aorta abdominal asociado a riñón en herradura: a propósito de dos casos y revisión de la literatura. Angiologia. 64(2):84-91. doi:10.1016/j.angio.2011.11.002.

Arquillo IML, Rey JV, Mu EF, Sá JME De, Rosendo A. Angiología. Angiologia. 2013;65(6):228-230.

Ezzet F, Dorazio R, Herzberg R. (1977). Horseshoe and pelvic kidneys associated with abdominal aortic aneurysms. Am J Surg. 134(2):196-198. doi:10.1016/00029610(77)90342-7.

Stroosma OB, Kootstra G, Schurink GW. (2001). Management of aortic aneurysm in the presence of a horseshoe kidney. Br J Surg. 88(4):500-9. doi:10.1046/j.13652168.2001.01718.x.

Ferko A, Krajina A, Jon B, Lesko M, Voboril Z. (1997). Juxtarenal aortic aneurysm associated with a horseshoe kidney. Transfemoral endoluminal repair. Arch Surg. 132(3):316-7. Available at: http://www.ncbi.nlm.nih.gov/pubmed/9 125035. Accessed July 3, 2015.

Brechtel K, Kalender G, Heller S, et al. (2009). Endovascular repair of infrarenal abdominal aortic aneurysm associated with a horseshoe kidney and graft thrombosis in early follow-up. Acta radiol. 50(6):624-628. doi:10.1080/02841850902902565.

Ruppert V, Umscheid T, Rieger J, et al. (2004). Endovascular aneurysm repair: Treatment of choice for abdominal aortic aneurysm coincident with horseshoe kidney? Three case reports and review of literature. J Vasc Surg Off Publ Soc 
Vasc Surg [and] Int Soc Cardiovasc Surgery, North Am Chapter. 40(2):367-370. doi:http://dx.doi.org/10.1016/j.jvs.2004. 04.014.

Gonzalez-Urquijo et al. (2021). EVAR in Patients With Abdominal Aortic Aneurysm and Horseshoe Kidney: A Systematic Review. Journal of Endovascular Therapy 00(0). doi/10.1177/15266028211059447

Sachsamanis et al. (2019). Management and therapeutic options for abdominal aortic aneurysm coexistent with horseshoe kidney. Journal of Vascular Surgery. Volume 69, Number 4 